\title{
Mathematisch-Naturwissenschaftliche Fakultät
}

Jean Christophe Tremblay | María Blanco-Rey

\section{Manipulating interfacial hydrogens at palladium via STM}

Suggested citation referring to the original publication:

Phys. Chem. Chem. Phys. 17 (2015), pp. 13973-13983

DOI http://dx.doi.org/10.1039/c5cp00663e

Postprint archived at the Institutional Repository of the Potsdam University in:

Postprints der Universität Potsdam

Mathematisch-Naturwissenschaftliche Reihe ; 291

ISSN 1866-8372

http://nbn-resolving.de/urn:nbn:de:kobv:517-opus4-99511 



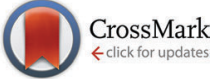

Cite this: Phys. Chem. Chem. Phys., 2015, 17, 13973

Received 2nd February 2015, Accepted 22nd April 2015

DOI: $10.1039 / \mathrm{c} 5 \mathrm{cp} 00663 \mathrm{e}$

www.rsc.org/pccp

\section{Manipulating interfacial hydrogens at palladium via STM}

\begin{abstract}
Jean Christophe Tremblay*a and María Blanco-Rey ${ }^{\text {bc }}$
In this contribution, we provide a detailed dynamical analysis of the interfacial hydrogen migration mediated by scanning tunneling microscopy (STM). Contributions from the STM-current and from the non-adiabatic couplings are taken into account using only first principle models. The slight asymmetry of the tunnelling rates with respect to the potential bias sign inferred from experimental observations is reproduced by weighting the contributions of the metal acceptor-donor states for the propagation of the impinging electrons. The quasi-thermal inelastic collision mechanism is treated perturbatively. The influence of hydrogen pre-coverage is also investigated using new potential energy surfaces obtained from periodic density functional theory calculations. Fully quantum dynamical simulations of the system evolution are performed by solving the Pauli master equation, providing insight into the reaction mechanism of STM manipulation of subsurface hydrogens. It is observed that the hydrogen impurity favors resurfacing over occupation of the bulk and subsurface sites whenever possible. The present simulations give strong indication that the experimentally observed protuberances after STM-excitation are due to hydrogen accumulating in the vicinity of the surface.
\end{abstract}

\section{Introduction}

Over the last three decades, scanning tunneling microscopy (STM) has become an indispensable characterization technique in surface science. Apart from the well-established imaging procedures, where surface topology can be characterized accurately, ${ }^{1,2}$ direct manipulation of adsorbed species in condensed environments has become possible. ${ }^{3}$ This includes applications ranging from bond breaking ${ }^{4,5}$ to conformational switching ${ }^{6-8}$ and vibrational excitations in adsorbates. ${ }^{9,10}$ In a few cases, the detection of impurities buried skin-deep below various surfaces was also achieved experimentally, along with the atomistic control of their motion. ${ }^{11-13}$

In a pioneering experiment, Sykes and co-workers demonstrated the possibility to address hydrogen at metallic interfaces using scanning tunneling microscopy. ${ }^{14}$ By varying the STM current and the applied potential bias, they were able to create protuberances on a $\operatorname{Pd}(111)$ surface in the low dilution limit. These were attributed to the migration of hydrogen atoms from the bulk palladium to the subsurface sites, causing the rise of the upper palladium layer observed in imaging mode.

\footnotetext{
${ }^{a}$ Institute for Chemistry and Biochemistry, Freie Universität Berlin, Takustr. 3, 14195 Berlin, Germany. E-mail: jc.tremblay@fu-berlin.de;

Fax: +49 30838 452051; Tel: +493083858150

${ }^{b}$ Departamento de Física de Materiales, Facultad de Químicas, Universidad del País Vasco UPV/EHU, Apartado 1072, 20080 Donostia-San Sebastián, Spain

${ }^{c}$ Donostia International Physics Center DIPC, P. Manuel de Lardizabal 4,

20018 Donostia-San Sebastián, Spain
}

A slight asymmetry in the protuberance height after STM manipulation was interpreted as a more efficient diffusion reaction at positive bias. This finding was explained in recent theoretical work by Blanco-Rey et al. ${ }^{15}$ where the propagation of impinging electrons causing the hydrogen migration was shown to follow an S-wave propagation mechanism in the bulk.

Further experimental work by Mitsui and co-workers ${ }^{16}$ demonstrated the effect of the STM-induced electric field on the patterning of atomic hydrogen on the same surface. Accompanying density functional calculations have shown that, for certain highly covered surfaces and at high electric field intensity, the population of subsurface sites could become thermodynamically more stable than in the zero field case. This trend was only observed at large positive bias. Nevertheless, the experiments demonstrated unambiguously that STM-induced electric fields can lead to a redistribution of the hydrogen at the interface, and that this redistribution is reversible. More importantly, the STM images after excitation showed large protrusions that were attributed to a local accumulation of interfacial hydrogens.

As presented elsewhere, ${ }^{17}$ we investigated the stability of hydrogen impurities in and at a $\operatorname{Pd}(111)$ surface from first principles to elucidate the effect of the STM-induced electric field on the thermodynamic equilibrium. It was found that the potential distortion due to the STM tip remains marginal for a wide range of potential biases. In particular, the reaction barriers for hydrogen diffusion at the interface were not found to be significantly affected by the STM field, which indicates that the reaction constant should remain almost invariant upon application of a bias voltage. 
On the contrary, the population dynamics of the different sites at the interface is affected by the presence of the STM tip. The nuclear dynamics of an impurity at metal surfaces is known to be induced by non-adiabatic coupling with the electrons of the environment. ${ }^{18-20}$ A perturbative model for computing rates associated with non-adiabatic coupling of an impurity embedded in a metallic environment was recently introduced. ${ }^{21}$ The model includes both energy relaxation/excitation rates due to electronhole pair creation/annihilation in the metallic environment, as well as the STM excitation mechanism. It is based on the evaluation of the vibronic coupling induced by the nuclear kinetic energy operator in the basis of one-electron Kohn-Sham states. The travelling electrons from/to the STM tip are treated in a sourcebridge-sink formalism, allowing for an efficient numerical resolution of the coupling matrix elements. The electronic contribution is found to be proportional to the density of states projected on the impurity.

It was observed from the rates that the mechanism for STM excitation at metals is of quasi thermal nature, a fact that was further emphasized by quantum dynamical simulations. The latter revealed that the hydrogen impurity exhibits a strong tendency towards resurfacing upon application of a potential bias, with no population left in the subsurface site at longer times. These investigations were limited to the low dilution case, without considering possible prior coverage of the palladium. Further, the metal surface was kept frozen, which might have affected the result for the relaxation rates, the transfer times, and the STM-driven dynamics. Finally, the original version of the non-adiabatic coupling rate model was symmetric with respect to the sign of the potential bias, with a projected density of states considered constant close to the Fermi energy. This is inconsistent with the experimental findings that show a slightly more efficient reaction at positive voltages.

In the present work, we extend the previous model to include the asymmetry of the non-adiabatic coupling rates with respect to the potential bias sign. To this end, we use the ideas proposed by Blanco-Rey et $a .^{15}$ to take into account the number of accessible metallic states for the travelling electrons. Further, we investigate the population dynamics of a hydrogen impurity in the vicinity of a palladium surface at different surface precoverages. The use of a Pauli master equation allows to treat the STM-induced dynamics and the relaxation/excitation due to electron-hole pair dynamics in the metal on an equal footing. From the simulations, the effect of surface pre-coverage on the transfer rates induced by STM excitations will be inferred. In the process, new potential energy surfaces including relaxation of the palladium and hydrogen layers are used, which will allow to evaluate the importance of the various contributions to the mechanism of STM-induced hydrogen diffusion at the interface.

The paper is organized as follows: the reaction path, the potential energy curves at different surface coverages, and the vibrational eigenstates of the model system are introduced in Section 2.1. The dynamical equations of motion and the nonadiabatic coupling rate expression for STM excitations at metallic surfaces are described in Section 2.2. The resurfacing dynamics, the transfer rates, and the equilibrium constants are investigated in Sections 3.1-3.3, respectively. Conclusions are presented in Section 4.

\section{Computational aspects}

\subsection{Model system}

We investigate the dynamics of a hydrogen impurity at a $\operatorname{Pd}(111)$ surface for various degrees of hydrogen pre-coverage. All potential energy curves for the atomic hydrogen impurity below a free and hydrogen-coated $\operatorname{Pd}(111)$ surface are calculated self-consistently using periodic density functional theory (DFT). Scalar relativistic ultrasoft pseudopotentials $\dagger$ and the PBE functional, ${ }^{22}$ as implemented in the Quantum Espresso package, ${ }^{23}$ are used for the simulations. To represent the $\operatorname{Pd}(111)$ surface, a $2 \times 2$ slab consisting of 6 layers of palladium (lattice constant: $3.98 \AA$ ) separated by a vacuum of $11.4 \AA$ is used. A $8 \times 8 \times 1$ Monkhorst-Pack grid ${ }^{24}$ with energy cutoff of $340.1 \mathrm{eV}$ and charge density cutoff of $3401 \mathrm{eV}$ are used in all calculations, and the energy is converged to $1.36 \times 10^{-7} \mathrm{eV}$. Since it was demonstrated that distortion of the potential energy surface by the STM tip is only marginal outside the surface and vanishing inside, ${ }^{17}$ the induced field is neglected throughout this work.

2.1.1 Reaction path. Different surfaces have been investigated along a one-dimensional reaction path: a clean $\operatorname{Pd}(111)$ surface, a saturated $\operatorname{Pd}(111)$ surface with all hcp sites occupied by hydrogen atoms, a saturated $\operatorname{Pd}(111)$ surface with all fcc sites occupied by hydrogen atoms, and the same surface with a hydrogen pre-coverage of $\Theta=3 / 4$ (see ref. 17). It was found that a reasonably accurate one-dimensional description of the reaction path is given by a sequence of $\mathrm{H}$ displacements perpendicular and parallel to the surface, which correspond to jumps from octahedral to tetrahedral sites, and conversely. For the resurfacing sections along the reaction path, the atomic hydrogen impurity is moved perpendicularly to the bottom of the slab, while the motion parallel to the surface is optimized for each configuration, along with the position of all palladium atoms of the five topmost layers, to provide a minimal energy path from the bulk to the surface. The discontinuities in the reaction path have been removed by extending the path with points parallel to the surface when leaving the octahedral cavity for the tetrahedral cavities, for which the perpendicular motion of the impurity has been optimized. The reaction path is depicted schematically as white beads in the inset of Fig. 1. Upon resurfacing, the hydrogen impurity is observed to displace the coating atom at the resurfacing fcc site, which diffuses towards the adjacent hcp site (not shown).

2.1.2 Potential energy curves. The electronic energy of different configurations of the atomic impurity inside and outside the palladium surface, interpolated using cubic splines, is reported in Fig. 1. In the remaining of this paper, the reaction path will be labeled after the symmetry of the nearest local

$\dagger$ The pseudopotentials "Pd.pbe-nd-rrkjus.UPF" and "H.pbe-rrkjus.UPF" from http://www.quantum-espresso.org are used for palladium and hydrogen, respectively. 


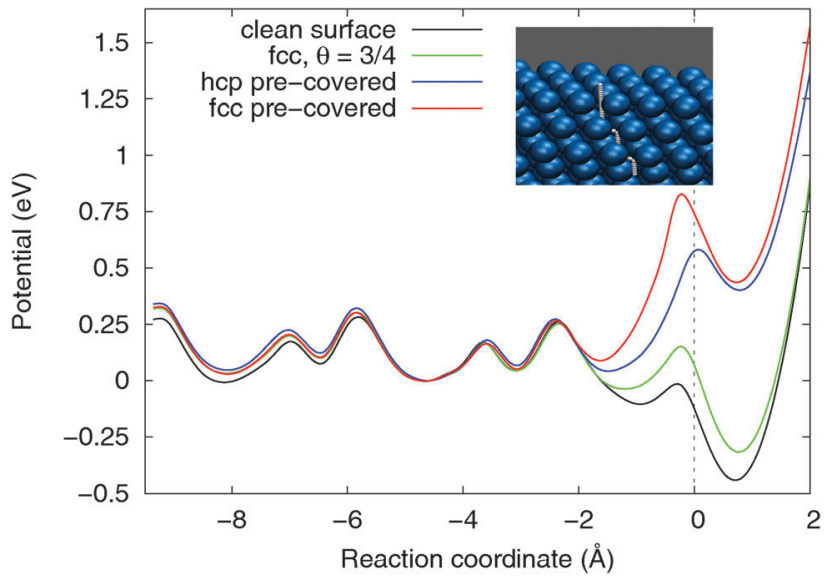

Fig. 1 Potential energy surfaces for the diffusion of hydrogen inside a $\operatorname{Pd}(111)$ surface. The reaction coordinate involves the motion of the hydrogen atom perpendicular and parallel to the surface. The surface zero is defined as the position of the topmost unrelaxed palladium layer. The absolute energy is shifted to adjust the second octahedral subsurface sites at the same energy. Inset: schematic representation of the diffusion of hydrogen inside a palladium surface. The blue balls represent palladium atoms and the white beads represent the migration of the hydrogen impurity along the reaction path (data from ref. 17).

minimum. From the bulk, the path begins in the octahedral site $\mathrm{O}_{\mathrm{b}}$ via the second subsurface octahedral site $\left(\mathrm{O}_{\mathrm{sb}}\right)$ to the octahedral subsurface site $\left(\mathrm{O}_{\mathrm{ss}}\right)$, before resurfacing at the fcc surface site (S). Each section of the path connecting two octahedral cavities is separated by tetrahedral sites, respectively labeled $\mathrm{T}_{\mathrm{b}}$ and $\mathrm{T}_{\mathrm{sb}}$. The following trends can be observed:

(1) For the clean surface, the impurity is most stable above the surface, and the $\mathrm{O}_{\mathrm{ss}}$ site is the most stable in the substrate.

(2) For both fully coated surfaces, the $\mathrm{O}_{\mathrm{sb}}$ site is the most stable.

(3) The local minima located in the octahedral cavities at about $-5.8 \AA\left(\mathrm{O}_{\mathrm{b}}\right),-3.5 \AA\left(\mathrm{O}_{\mathrm{sb}}\right)$, and $-1.3 \AA\left(\mathrm{O}_{\mathrm{ss}}\right)$ are more stable than those located in the tetrahedral cavities at about $-5.1 \AA$ $\left(\mathrm{T}_{\mathrm{b}}\right)$ and $-3.0 \AA\left(\mathrm{T}_{\mathrm{sb}}\right)$.

(4) The barrier to resurfacing is large for all coated surfaces.

(5) Relaxing the position of the substrate reduces significantly the diffusion barrier. ${ }^{25,26}$

The barrier to resurfacing for the rigid clean surface (273 meV) is somewhat higher than that of previous calculations $(198 \mathrm{meV}) .{ }^{27}$ The discrepancies could be due to the choice of pseudopotentials and other convergence parameters. In particular, the sampling of the Brillouin zone is here much higher $(8 \times 8 \times 1$ vs. $4 \times 4 \times 1)$, whereas the energy cutoff for the plane wave basis is much lower (340.1 eV vs. $816.34 \mathrm{eV})$. This should only affect the observed trends qualitatively. More details concerning the static DFT calculations are given elsewhere. ${ }^{17}$

2.1.3 Vibrational eigenstates. The vibrational eigenstates of the hydrogen impurity are computed variationally. The system is described by the following one-dimensional Hamiltonian

$$
\hat{H}_{1 \mathrm{D}}(q)=-\frac{\hbar^{2}}{2 m_{q}} \frac{\mathrm{d}^{2}}{\mathrm{~d} q^{2}}+V(q)
$$

where $m_{q}$ is the mass of the hydrogen impurity and the potential $V(q)$ is computed as described in the previous section. The generic coordinate $q$ is chosen to emphasize the fact that the reaction path has portions perpendicular and parallel to the surface. The Hamiltonian is represented in a basis of 500 sinc discrete variable representation functions on the range $[-10 \AA$, $2 \AA]$. An in-house implementation of the coupled two-term Lanczos algorithm with full reorthogonalization ${ }^{28,29}$ is used to extract the low-lying eigenstates of the Hamiltonian matrix below $1.240 \mathrm{eV}$ above the potential energy minimum. The number of states found is of $96,97,91$, and 89 for the clean, $\Theta=3 / 4$, hcp pre-covered, and fcc pre-covered surfaces, respectively.

The lowest states are observed to be localized in the octahedral cavities along the reactions path, with the degree of delocalization increasing close to the diffusion barriers. The shallow and narrow diffusion barriers from the tetrahedral sites do not support localized bound states in most cases and the vibrational wave functions can be assimilated to Fermi resonances above the neighboring octahedral minimum. The states above the bulk diffusion barrier are strictly delocalized inside the surface. It is important to recognize that the choice of the integration boundaries imposes a node at $q=-10 \AA$ and $q=2 \AA$, which is not physical for the lower boundary. Excluding the surface bound states supported by both pre-covered surfaces, all delocalized states above the bulk diffusion barrier and up to the vibrational energy cutoff $\left(E_{\text {cut }}=1.240 \mathrm{eV}\right)$ should thus be regarded as a discretized version of a continuum of states inside the metal. Whereas these states are important for driving the dynamics, they are only marginally populated at any given time during the STM transfer process. They should thus be considered merely as a computational basis, and the number included in the dynamical simulations should be and has been converged variationally.

\subsection{Equations of motion}

As in previous work, ${ }^{21}$ the population evolution of the system states driven by the STM is studied using the Pauli master equation

$$
\frac{\mathrm{d} P_{n}(t)}{\mathrm{d} t}=\sum_{j}\left(\Gamma_{j \rightarrow n} P_{j}(t)-\Gamma_{n \rightarrow j} P_{n}(t)\right),
$$

where $P_{n}(t)$ is the population of state $n$. By doing so, we choose to neglect the coherences between the system eigenstates. This choice is physically sound since the STM-induced dynamics proceeds on a very long time scale ( $>$ nanosecond) compared to dephasing in metallic environments (sub-picosecond). The dynamical basis is obtained from the diagonalization of the one-dimensional reaction path Hamiltonian as described in the previous section. The dynamics is driven by the various transition rates from states $i$ to $j, \Gamma_{i \rightarrow j}$. For dynamics at metal surfaces, the vibrational coupling of the impurity to the electronhole pairs of the metallic environments is known to dominate. Whereas explicit treatment of vibration-phonon coupling is neglected here, a large part of the deformation potential is taken 
into account by the adiabatic relaxation of the palladium atoms along the reaction path.

2.2.1 Relaxation/excitation rates. The transfer rates due to electron-hole pair coupling are computed using a recently published anharmonic rate model. ${ }^{21}$ For a single vibrational mode $q$, the total upward and downward transition rates, including the zero-bias relaxation and the STM-induced transitions, are calculated according to $\left(\omega_{i j}>0\right)$

$$
\begin{aligned}
\Gamma_{i \rightarrow j} & =\left(1+B\left(\hbar \omega_{i j}\right)+w_{\mathrm{s}}(I, U)\left(\frac{|e U|}{\hbar \omega_{i j}}\right)\right) \gamma_{i j}^{q}, \\
\Gamma_{i \leftarrow j} & =\left(B\left(\hbar \omega_{i j}\right)+w_{\mathrm{s}}(I, U)\left(\frac{|e U|}{\hbar \omega_{i j}}\right)\right) \gamma_{i j}^{q} .
\end{aligned}
$$

Here, $U$ is the potential bias, $I$ is the total STM current, and $\omega_{i j}$ is the transition frequency. Further, the Bose-Einstein distribution accounts for temperature effects

$$
B\left(\hbar \omega_{i j}\right)=\frac{1}{\mathrm{e}^{\hbar \omega_{i j} / K_{\mathrm{b}} T}-1}
$$

The rates increase with temperature and obey the principle of detailed balance at zero bias. In the present work, the temperature is left constant at $T=0 \mathrm{~K}$. The non-adiabatic coupling factor is given by

$$
\gamma_{i j}^{(q)}=\frac{\gamma^{(q)}}{\omega_{i j}}\left|\left\langle j\left|\frac{\partial}{\partial q}\right| i\right\rangle\right|^{2}, \gamma^{(q)}=\left(\frac{2 \hbar \Gamma^{(\mathrm{ref}, q)}}{m_{q}}\right) .
$$

with $m_{q}$ the mass of the atomic impurity and $\Gamma^{(\mathrm{ref}, q)}$ a reference transition rate. A value of $1 / \Gamma^{(\mathrm{ref}, q)}=400 \mathrm{fs}$ was chosen in all simulations, in accord with previous estimates of the perpendicular and parallel mode vibration lifetimes, ${ }^{30}$ which were found to be similar inside and outside the surface. The present electron-hole pair coupling model does not include variation of the embedding density as a function of the position to simplify comparison between the different surfaces. Below the first palladium layer $(q<0)$, the embedding density varies in the range $r_{\mathrm{s}} \in[2,2.5] a_{0}$ for the clean surface, and the value for the fully covered surfaces, e.g. for the hcp $-\Theta=1$ case, is equal to within $0.05 a_{0}$. At the surface, $q>0$, the embedding density values drop to $r_{\mathrm{s}}=4 a_{0}$. In this region, the $r_{\mathrm{s}}$ of the clean slab is also found to be $\sim 0.4$ larger than that of the saturated slab, which could have a minor impact on the transition rates. As will be shown below, the hydrogen diffusion dynamics is not strongly affected by short-time relaxation, as the former happens on a much longer timescale and the latter merely ensures that the system remains in quasi-thermal equilibrium at any given time.

The prefactor $w_{\mathrm{s}}$ represents the relative efficiency of the STM coupling mechanism, which can be related to the ratio of projected densities of states (PDOS) from the STM tip and the substrate on the impurity. ${ }^{18-21}$ The PDOS is in turn related to the electron density of the metallic surroundings via an embedding atom model, where the impinging electrons are hopping from one palladium atom to the other. In previous work, we used the PDOS computed using periodic DFT to evaluate the S-wave contribution to the rates, as it was demonstrated to be the driving force for hydrogen diffusion inside the bulk. This is likely to be exact deep below the surface but this approximation could more strongly affect the dynamics at the interface. Instead, we choose here to take into account all electrons of the embedding environment. Following Blanco-Rey and co-workers, ${ }^{15}$ the STM prefactor of the previous model ${ }^{21}$ is thus modified to take into account the current-dependence of the STM-induced rates

$$
w_{\mathrm{s}}(I, U)=\frac{I \tau_{0}}{e \rho_{0}(U)} .
$$

The residence time $\tau_{0}$ of the electron on the impurity is chosen equal to the reference lifetime of the $q$-mode vibration lifetime, $\tau_{0}=1 / \Gamma^{(\mathrm{ref}, q)}=400 \mathrm{fs}$. The function $\rho_{0}(U)$ represents the number of available states per palladium atom for the STM electrons at a given potential bias. At positive (negative) bias, these are the empty (occupied) one-electron states of the metal (see inset of Fig. 4(b)). It is found that, for the system at hand, the function is slightly asymmetric with respect to the potential bias sign, which is consistent with the experimental observations by Sykes and co-workers ${ }^{14}$ from which the transfer rates are inferred. For more information on the rate model, the reader is referred to the relevant literature. ${ }^{15,21}$

\section{Results and discussion}

\subsection{Resurfacing dynamics}

The STM excitation dynamics at $0 \mathrm{~K}$ is investigated over a moderate potential bias range of $U=[-1,1] \mathrm{V}$ and in the weak current limit, $I \in\{100,200,500,1000\}$ pA. Given the low temperature of the experiment, no significant thermal population of higher excited states is expected. The energy of various octahedral sites relative to the initial state is given in Table 1 for different surface coverages. First, it appears that the effect of the hydrogen layer can be felt very deeply in the surface. Whereas the deeper wells behave similarly for all pre-covered surface, independent of the coverage, the subsurface is more

Table 1 Energy of octahedral sites relative to the second subsurface state in the bulk $\left(E_{i}\right)$ and associated diffusion barriers $\left(\Delta E^{*}\right)$, as a function of the hydrogen pre-coverage. All values in meV

\begin{tabular}{lrrrr}
\hline & Clean & fcc, $\Theta=3 / 4$ & fcc, $\Theta=1$ & hcp, $\Theta=1$ \\
\hline$E_{\mathrm{S}}$ & -443 & -312 & 442 & 403 \\
$\Delta E_{\mathrm{S} \rightarrow \mathrm{O}_{\mathrm{ss}}}^{\ddagger}$ & 424 & 465 & 369 & 168 \\
$\Delta E_{\mathrm{O}_{\mathrm{ss}} \rightarrow \mathrm{S}}^{\ddagger}$ & & & & \\
$E_{\mathrm{O}_{\mathrm{s}}}$ & -105 & -36 & 721 & 528 \\
$\Delta E_{\mathrm{O}_{\mathrm{ss}} \rightarrow \mathrm{O}_{\mathrm{sb}}}^{\ddagger}$ & 363 & 285 & 90 & 43 \\
$\Delta E_{\mathrm{O}_{\mathrm{sb}} \rightarrow \mathrm{O}_{\mathrm{ss}}}^{\ddagger}$ & 258 & 249 & 175 & 227 \\
$E_{\mathrm{O}_{\mathrm{sb}}}$ & 0 & 0 & & \\
$\Delta E_{\mathrm{O}_{\mathrm{sb}} \rightarrow \mathrm{O}_{\mathrm{b}}}^{\ddagger}$ & 272 & 289 & 265 & 271 \\
$\Delta E_{\mathrm{O}_{\mathrm{b}} \rightarrow \mathrm{O}_{\mathrm{sb}}}^{\ddagger}$ & 278 & 254 & 0 & 0 \\
$E_{\mathrm{O}_{\mathrm{b}}}$ & -6 & 35 & 289 & 309 \\
& & & & 260
\end{tabular}


sensitive to surface modification. The fully covered surfaces show a slight preference for the second subsurface site, while the impurity seems to prefer the surface site whenever it is not fully saturated. Resurfacing is found to be a thermodynamically activated process for a $\Theta=3 / 4$ pre-coverage of the fcc sites but not for the clean surface. Further, the hcp pre-covered surface has almost isoenergetic sites on each side. Upon excitation, the system thus should exhibit a slight preference towards the subsurface because of the smaller diffusion barrier. To investigate the preference of the impurity towards either resurfacing or bulk diffusion, the dynamics is initiated in the second subsurface octahedral site $\left(\mathrm{O}_{\mathrm{sb}}\right)$. The latter is similar in shape and curvature to the bulk site but still feels the influence of surface coverage partially ( $c f$. Fig. 1 and Table 1). Fig. 2(a)-(c) show the population evolution of the ground states associated with each well for varying coverage and under different STM conditions. Similar population dynamics can be seen for both fully covered surfaces and only the hcp $-\Theta=1$ results are shown. The population of the vibrational excited states in the initial well is also represented (label "transient"). For the low current cases (left panels), the dynamics is seen to happen on a very long time scale but an exponential behaviour can be clearly recognized. The dynamics at higher current (right panels) exhibits the same trends and is seen to reach a quasi equilibrium for all simulations within a few tens of ns. It is important to note that no time-resolved experimental results are available, but the timescales presented here are well below the experimental upper limit of a few minutes. Hence, the observed protuberances $^{14}$ should be considered as the asymptotic limit of the population evolution upon STM excitation. The depopulation of the initial state is quantitative for the unsaturated surfaces, while it remains far from quantitative for the saturated ones. This correlates well with the data in Table 1 and the quasi-thermal STM excitation mechanism, which predicts that the most stable species in the saturated cases is the second subsurface octahedral site. Although some population tunnels from the initial state to the neighboring minima, the bulk of the population remains in the initial configuration.

Taking the ratio of excitation and relaxation rates in eqn (2) yields the following skewed distribution at equilibrium

$$
R_{i j}=\frac{\Gamma_{i \leftarrow j}}{\Gamma_{i \rightarrow j}}=\frac{B\left(\hbar \omega_{i j}\right)+w_{\mathrm{s}}(I, U)\left(\frac{|e U|}{\hbar \omega_{i j}}\right)}{1+B\left(\hbar \omega_{i j}\right)+w_{\mathrm{s}}(I, U)\left(\frac{|e U|}{\hbar \omega_{i j}}\right)}
$$

which is independent of the non-adiabatic coupling factor, eqn (3). The intermediate population of the transient states emphasizes the nature of the excitation mechanism: the initial state is moved out of its thermal equilibrium after the STM perturbation is turned on. The dynamics is driven by the population of vibrationally excited states to reach a skewed thermal distribution. The probability of tunneling through the barrier is larger at higher energy and so is the probability of above-threshold diffusion. The atoms reaching the other side of the barrier swiftly relax to a local quasi-thermal distribution determined by eqn (5). In the process, other transient states (not depicted in Fig. 2(a)-(c)) are also populated and can mediate the next diffusion step.

The time evolution of the density projected on the reaction path is depicted in Fig. 3(a)-(d). The density is computed as the incoherent sum of state densities,

$$
\rho(q, t)=\sum_{n} P_{n}(t)\left|\psi_{n}(q)\right|^{2}
$$

where the wave functions are solution to the one-dimensional vibrational Schrödinger equation, $\hat{H}(q) \psi_{n}(q)=E_{n} \psi_{n}(q)$. The density provides information on the degree of localization of the hydrogen impurity in the different wells, as well as the eventual nodal structure in each well. The STM conditions are chosen as $I=1000 \mathrm{pA}$ and $U=0.7 \mathrm{~V}$, and the initial density is found in the second octahedral subsurface cavity. As was seen in the population dynamics, the impurity migrates preferentially to the surface sites when the surface is not saturated. Also, all but the surface with $\Theta=3 / 4$ hydrogen coverage have nonnegligible population in the bulk site at longer times, and the subsurface site gets only populated in the case of pre-coverage of the hcp sites. Further, the impurity moves preferentially towards the bulk for the fcc pre-covered surface, while the hcp pre-covered surface exhibits no strong preference towards the bulk or the subsurface. Consequently, both are about equally populated at the end of the propagation and much less than the initial site. These observations correlate again well with classical diffusion barriers and the relative energies reported in Table 1. It is interesting to note that the resurfacing dynamics for the $\Theta=3 / 4$ surface happens on a faster timescale as for the clean surface, which could be observed to a lesser extent in the population dynamics, Fig. 3(a)-(d). The rationale is that the bulk octahedral site is more stable than the second subsurface site for the clean surface but the reverse is true for the $\Theta=3 / 4$ surface. The resurfacing dynamics thus happens directly for the latter, whereas it can take a detour via the bulk in the former case. At longer times, the bulk population is found to also decay exponentially, as the surface state ultimately attracts the hydrogen impurity. Finally, although the hydrogen transfer happens via excitation of transient vibrational states (see Fig. 2(a)-(c)), no local nodal structure can be recognized in each well at any time during the dynamics. This is due to the fact that the transient species are short-lived and their population remains modest at all times.

\subsection{Population transfer}

As discussed in earlier work, ${ }^{21}$ the system first responds to the STM perturbation within a few hundreds of femtoseconds. This response time is almost independent of the initial conditions and varies linearly with the potential bias. By abruptly switching on the STM field, it can be efficiently decoupled from the STM-induced transfer time, which is the one that provides meaningful insight in the experiment. The transfer time $\tau_{t}$ can be obtained by fitting the population decay of the initial site, excluding the initial stages of the propagations, according to

$$
P_{i}(t)=P_{i}(\infty)+\mathrm{d} P_{i} \exp \left(-t / \tau_{t}\right)
$$



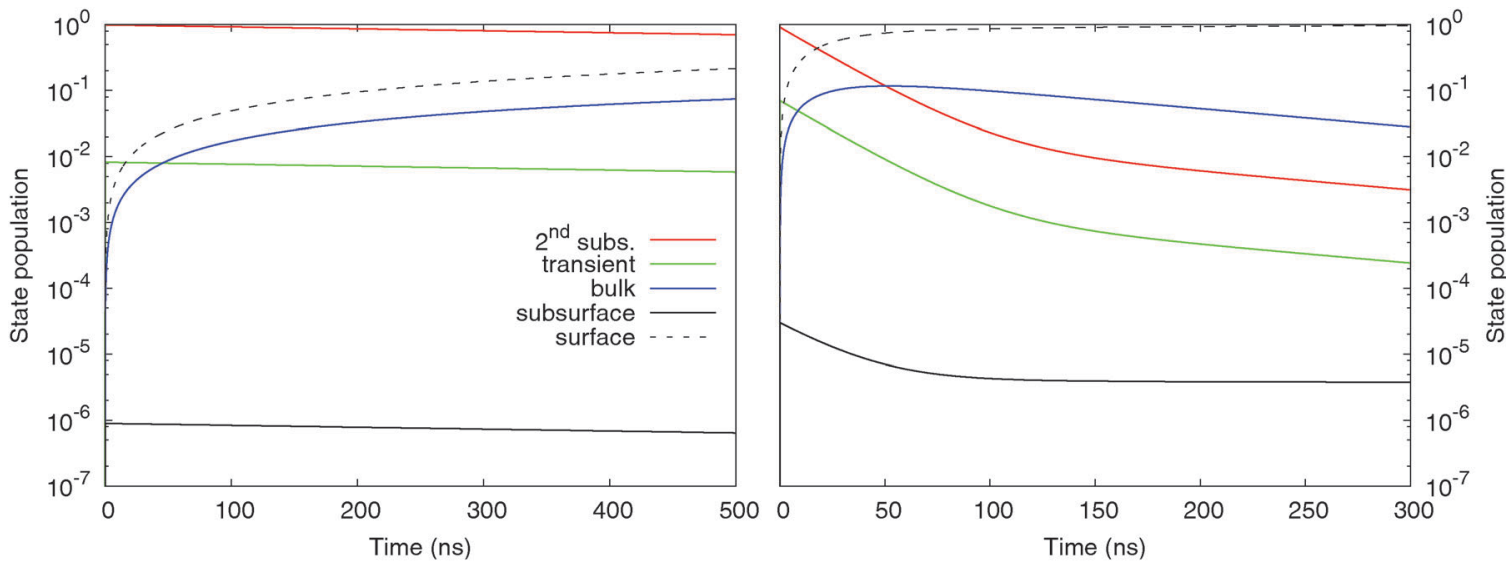

(a) clean surface
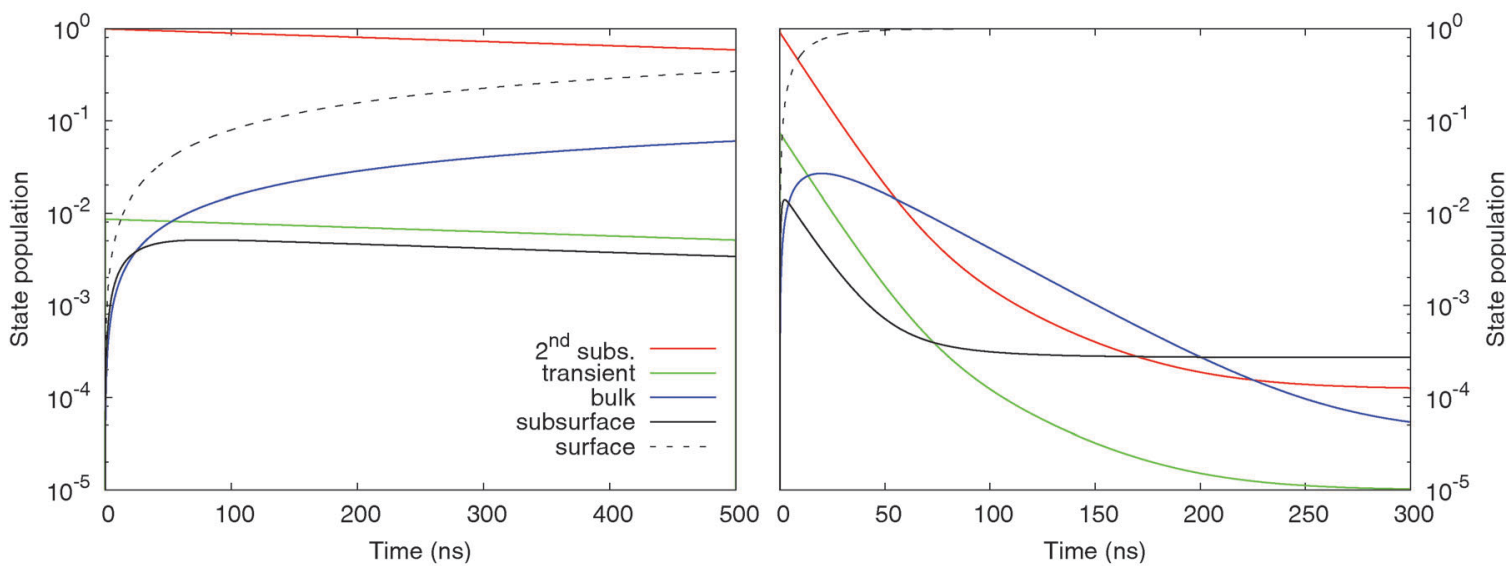

(b) fcc pre-covered, $\Theta=3 / 4$
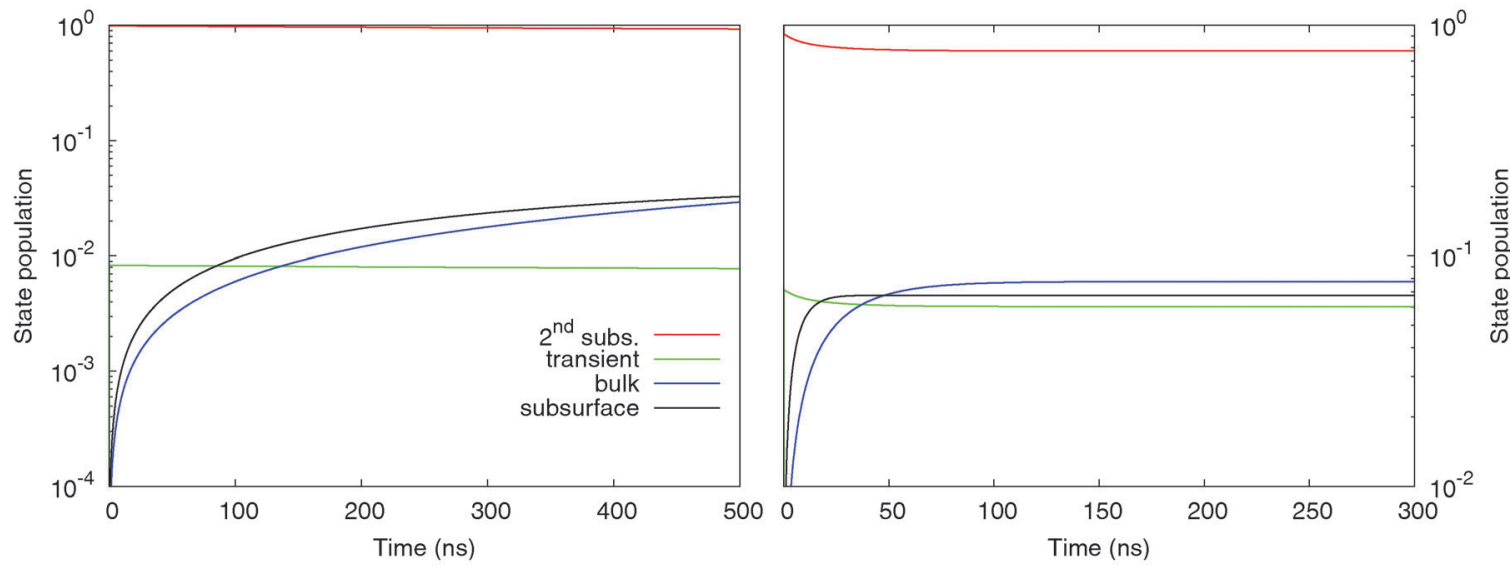

(c) hep pre-covered, $\Theta=1$

Fig. 2 Population dynamics of an atomic hydrogen impurity inside a $\mathrm{Pd}(111)$ surface driven by STM, at various hydrogen coverages. The population is initially placed in the second subsurface octahedral site $\left(\mathrm{O}_{\mathrm{sb}}\right)$. For both unsaturated surfaces, all the hydrogen impurities segregate to the surface site asymptotically. Left panels: $I=100 \mathrm{pA}, U=0.7 \mathrm{~V}$. Right panels: $I=1000 \mathrm{pA}, U=0.7 \mathrm{~V}$.

where $\mathrm{d} P_{i}$ is the transfer efficiency and $P_{i}(\infty)$ is the asymptotic population of the system. Fig. 4(a) and (b) show the dependence of the transfer time on the STM current and the potential bias. From the figures, we can observe that the current-dependence of the transfer time is approximately exponential at higher currents. Further, the transfer time dependence on the potential bias strongly departs linearity, as one would expect from eqn (2) and (4). This is due to the fact that, at negative bias, more states are available per palladium atom for the electron transfer. The quantity $\rho_{0}$ being higher, the transfer 


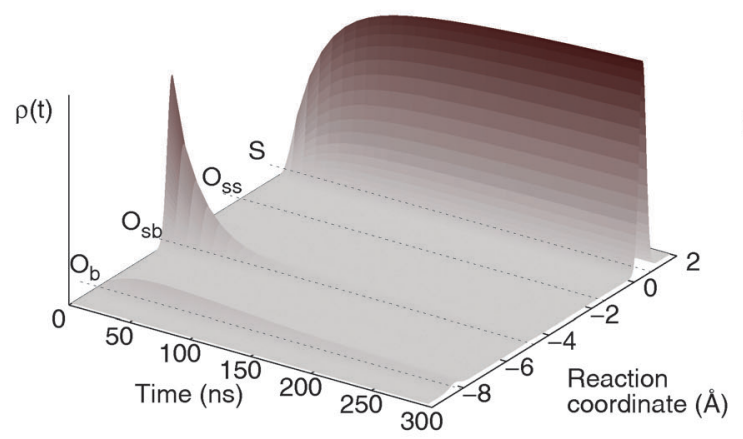

(a) clean surface

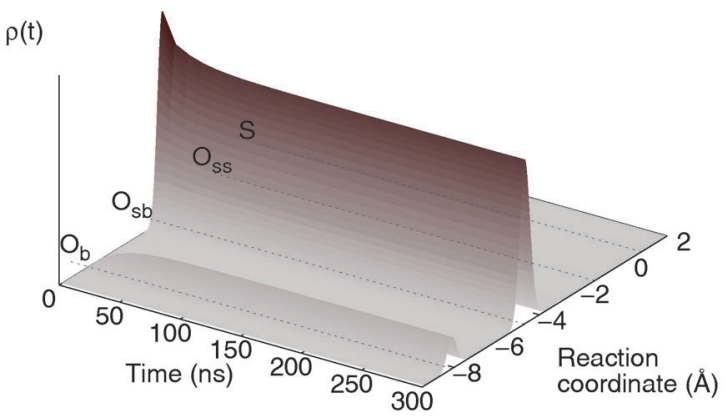

(c) fcc pre-covered, $\Theta=1$

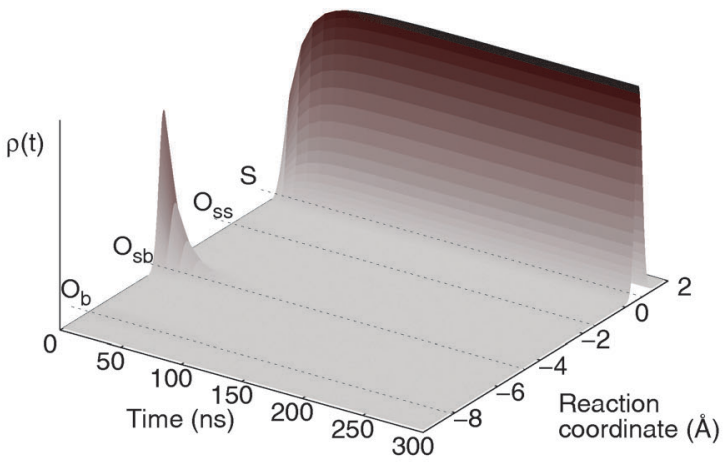

(b) fcc pre-covered, $\Theta=3 / 4$

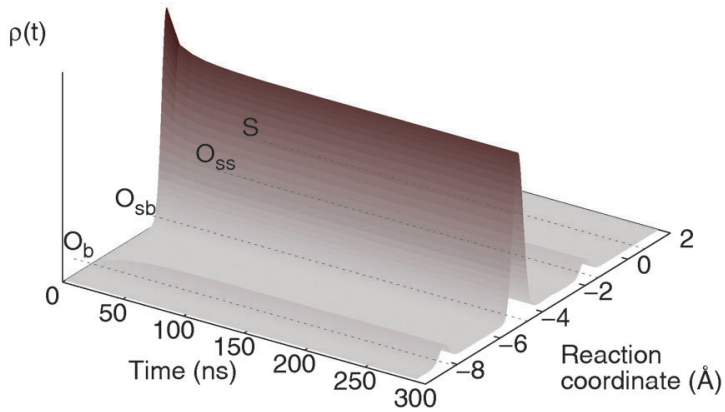

(d) hcp pre-covered, $\Theta=1$

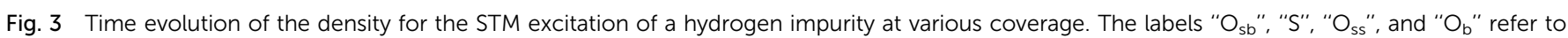

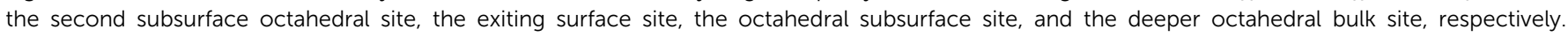
STM parameters: $I=1000 \mathrm{pA}, U=0.7 \mathrm{~V}$.

rates diminish (see eqn (4)) and the transfer times increase. Interestingly, the transfer time seems to be only marginally affected by surface coverage. In all simulations, both the transfer times and the inverse non-adiabatic coupling rates are found to be orders of magnitudes smaller than the inverse rates reported in previous work, ${ }^{15}$ indicating a much faster dynamics. The inverse excitation/deexcitation rates were previously found to be on the order of 2-100 ps (see Fig. 3 and the definition of $\Gamma_{0}$ below eqn (3) in ref. 15). This value is about 1-3 orders of magnitude longer than the one used in this work, $1 / \Gamma^{(\mathrm{ref}, q)}=400 \mathrm{fs}$, which was obtained from previous theoretical calculations. ${ }^{30}$ Accordingly, the transfer times reported in Fig. 5 are also about 2 orders of magnitude shorter than those reported in the work of Blanco-Rey et al. ${ }^{15}$ Note that both estimates are well below the experimental lower bound of minutes. More importantly, they exhibit the same trends with respect to the sign of the potential bias. It can be inferred that, since the rates are larger, the experimentally observed protuberances should be more pronounced for positive biases, which is indeed the case. ${ }^{14}$

From eqn (2) and (4), it appears that the transfer rate could be naturally recast as a function of an effective potential bias,

$$
U_{\text {eff }}=\left(\frac{I \tau_{0}}{e \rho_{0}}\right)|U|
$$

Although this variable is not experimentally measurable, it is instructive to look at the transfer times (i.e. the inverse transfer rates) as a function of $U_{\text {eff. }}$ It also allows to compare the transfer times for different potential biases and STM currents at once. From Fig. 5, we see that the transfer times have indeed a simple exponential dependence in the new variable. The low effective bias regime corresponds to the weak current limit $(I=\{100,200\} \mathrm{pA}, U=0.7 \mathrm{~V})$. The next two points belong to the large negative voltage case $(U=\{-0.6,-1.0\} \mathrm{V}, I=1000 \mathrm{pA})$. This is because the density of acceptor states per palladium atom, $\rho_{0}$, is larger at negative biases. This causes the times in eqn (2) to increase. The next value is associated with an intermediate current $(I=500 \mathrm{pA})$ and a positive bias $(U=0.7 \mathrm{~V})$. The remaining points have a larger current $(I=1000 \mathrm{pA})$ and increasing potential bias $(U=\{0.6,0.7,1.0\} \mathrm{V})$. As was observed for the current and bias dependence, the times associated with the $\Theta=3 / 4$ coverage appear to be a bit longer than the others, although only marginally. This trend can be related to the height of the activation barriers towards the bulk and the subsurface sites. Nonetheless, the slight preference for diffusion towards the bulk or the subsurface deeper inside the surface should be considered within the error tolerance of the underlying DFT/PBE potential energy surfaces.

The appearance of protuberances on the palladium surface can be correlated not only to the transfer times but also to the 


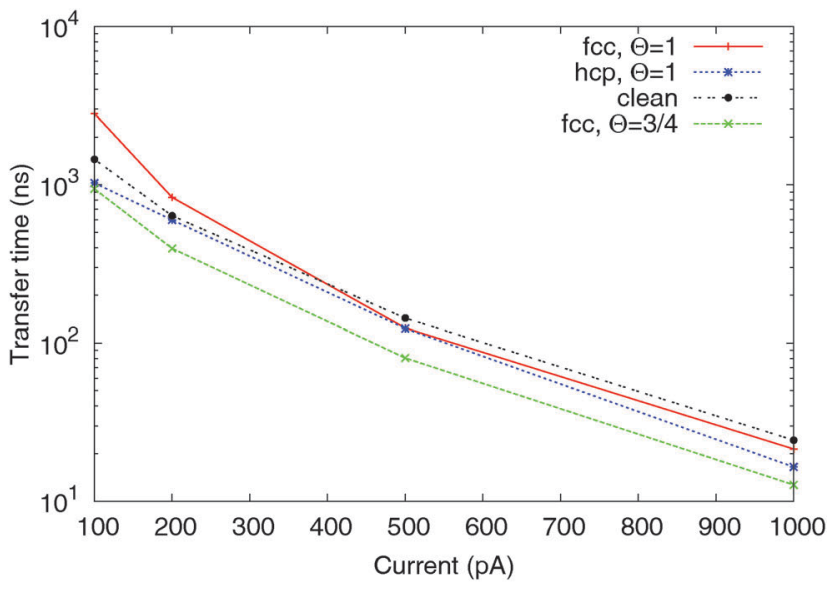

(a) Variable current at fixed potential bias, $U=0.7 \mathrm{~V}$.

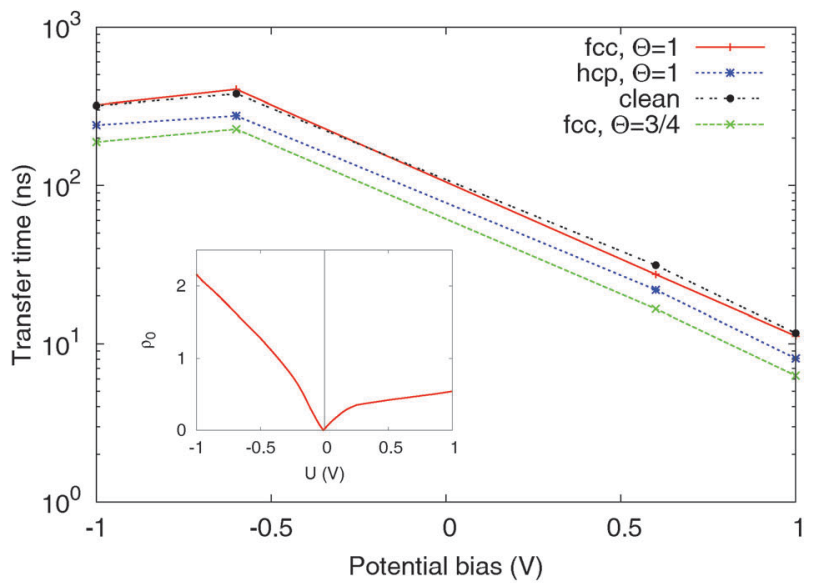

(b) Variable potential dependence at fixed STM current, $I=1000 \mathrm{pA}$.

Fig. 4 Dependence of the transfer time calculated fixing (a) the potential bias and (b) STM current for different hydrogen coverages of the palladium surface. The transfer time decreases exponentially with the current. The inset in panel (b) shows the number of available states per palladium atom as a function of the potential bias. The transfer time exhibits a slight asymmetry at large negative biases due to the increasing number of available palladium states.

transfer efficiencies, see eqn (6). The latter are depicted in Fig. 6 for the different coverages. For the unsaturated surfaces (top panel), the transfer from the second subsurface octahedral site is almost quantitative and diminishes at larger effective bias. This is because at higher effective bias, a larger fraction of the impurities are excited above the barrier. Incidentally, this barrier is also the largest to overcome in the system (see Fig. 1) and the excited hydrogen atoms can be pictured as more delocalized everywhere in and at the surface. Hence, a slight reduction in the transfer efficiency with increasing effective bias corresponds to a more democratic redistribution of the hydrogens upon relaxation. Note that the bulk of the population is found at the surface (see Fig. 2(a), (b) and 3(a), (b)), which contributes in part to the observed protuberances. For the saturated surfaces, the low-effective bias region is seen to depart strongly from linearity. This seems to indicate that, in this regime, tunneling through the barrier dominates. Further, the dip

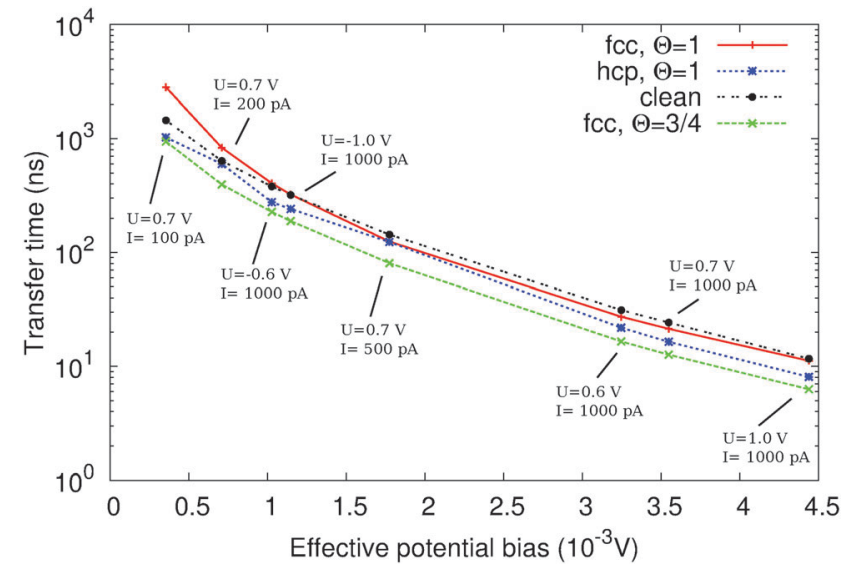

Fig. 5 Dependence of the transfer time on the effective potential bias, eqn (7). The transfer time is seen to decay approximately exponentially with $U_{\text {eff. }}$ The low-current regime is followed by a moderate current, large negative bias region, and by a high-bias region. See text for more details.

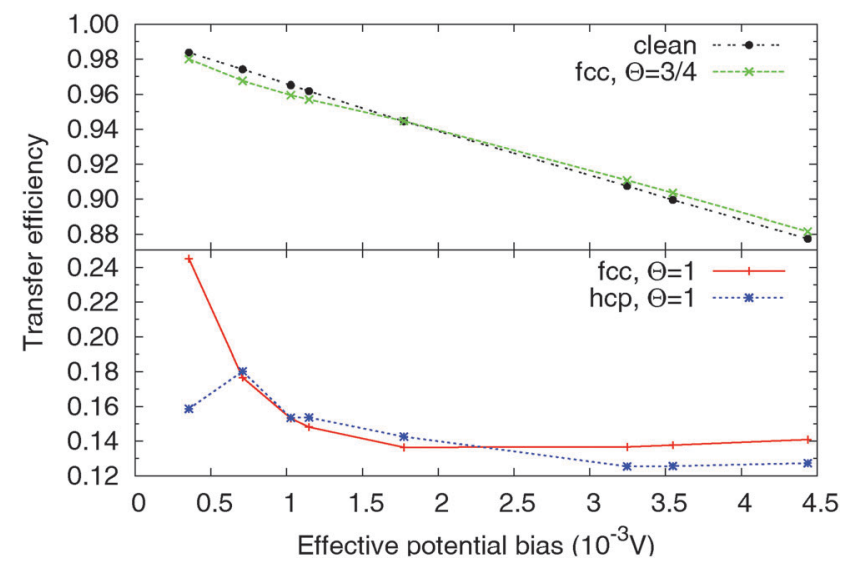

Fig. 6 Dependence of the transfer efficiency on the effective potential bias, eqn (7). The transfer is quantitative and scales linearly with $U_{\text {eff }}$ for partially covered surfaces (top panel). The saturated surfaces (bottom panel) allow only for a partial population transfer, as the second subsurface octahedral site is the thermodynamically most stable.

in efficiency at low bias for the hcp pre-covered surface is an indication of the system's preference for the second subsurface octahedral site, as shall be discussed in the next section. In the long time limit, part of the population is moved to both the bulk and the subsurface sites, with only the latter contributing to the rise of the palladium surface observed experimentally. It is important to note that, although some population is found in the subsurface site (see Fig. 3(c) and (d)), most will remain in the second subsurface site. This is in stark contrast to the population dynamics exhibited by the system upon excitation initiated from the subsurface site. In the top panel of Fig. 7, the subsurface population is seen to decay within a nanosecond to the second subsurface octahedral site due to the repulsion from the fcc hydrogen pre-covered surface. At longer times, the second subsurface population diffuses further to the bulk. A similar population dynamics is observed for the hcp pre-covered surface, albeit on a longer timescale. This observation correlates well with 


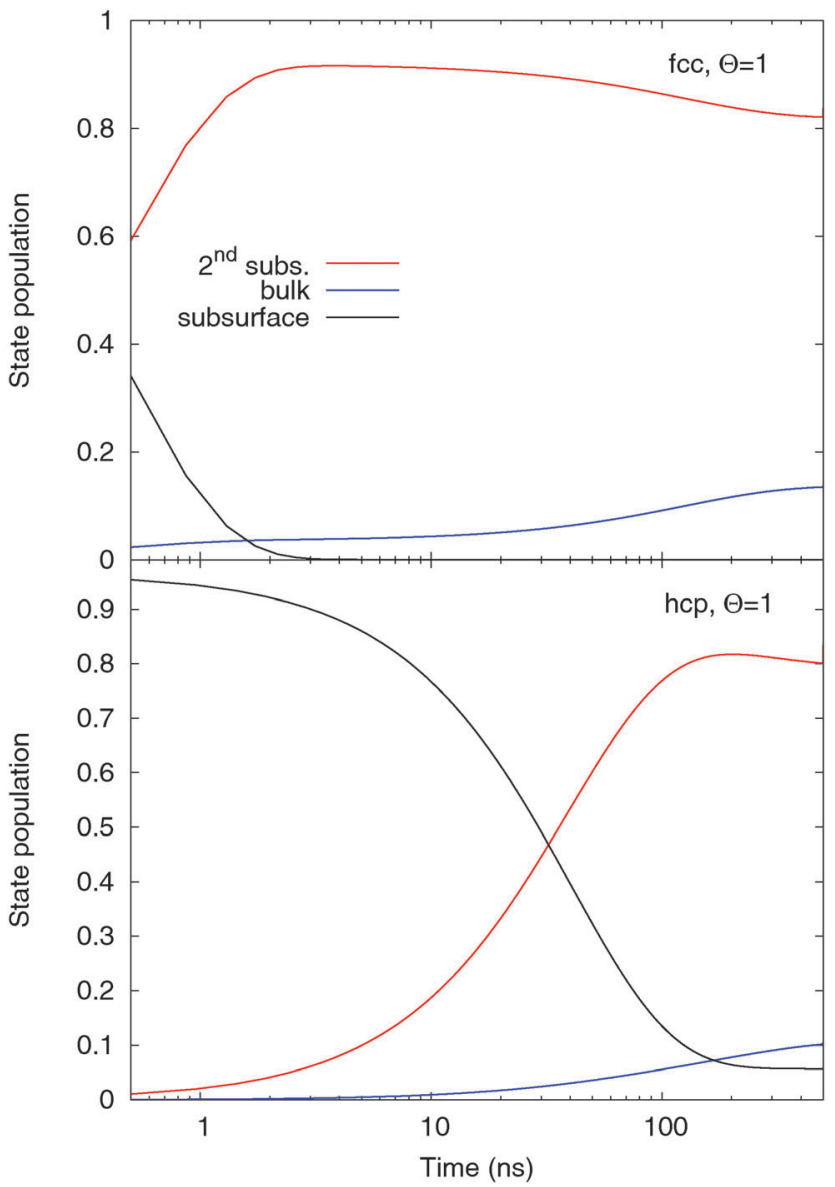

Fig. 7 Population dynamics of an atomic hydrogen impurity inside a $\mathrm{Pd}(111)$ surface driven by STM. The population is initially found in the subsurface octahedral site (top panel: fcc pre-covered, $\Theta=1$; bottom panel: hcp pre-covered, $\Theta=1$ ). The hydrogen impurity diffuses rapidly to the second subsurface STM parameters: $I=500 \mathrm{pA}, U=0.7 \mathrm{~V}$.

the barrier heights and the relative stability of the different octahedral sites. All dynamical simulations provide strong indication that subsurface hydrogen are not responsible for the experimentally observed rise of the palladium surface. On the contrary, they suggest that part of the observed protuberances are due to resurfacing of the hydrogen forming a coating layer, with further displacement of the surface due to the subsequent population of the deeper, thermodynamically most stable second subsurface site. The sole population of the subsurface site without prior saturation of the surface can be safely rejected as a mechanistic hypothesis.

\subsection{Equilibrium constants}

As argued above, the correlation between the transfer times and the diffusion barriers stems from the quasi-thermal nature of the STM excitation mechanism at metallic surfaces. The reaction path from the octahedral bulk site $\left(\mathrm{O}_{\mathrm{b}}\right)$ to the surface via the second and first subsurface octahedral sites $\left(\mathrm{O}_{\mathrm{sb}}\right.$ and $\mathrm{O}_{\mathrm{ss}}$, respectively) can be written schematically as

$$
\left(\mathrm{O}_{\mathrm{b}}\right) \underset{k_{\mathrm{O}_{\mathrm{sb}} \rightarrow \mathrm{O}_{\mathrm{b}}}}{\stackrel{k_{\mathrm{O}_{\mathrm{b}} \rightarrow \mathrm{O}_{\mathrm{sb}}}}{\rightleftharpoons}}\left(\mathrm{O}_{\mathrm{sb}}\right) \underset{k_{\mathrm{O}_{\mathrm{ss}} \rightarrow \mathrm{O}_{\mathrm{sb}}}}{\stackrel{k_{\mathrm{O}_{\mathrm{sb}} \rightarrow \mathrm{O}_{\mathrm{ss}}}}{\rightleftharpoons}}\left(\mathrm{O}_{\mathrm{ss}}\right) \underset{k_{\mathrm{S} \rightarrow \mathrm{O}_{\mathrm{ss}}}}{\stackrel{k_{\mathrm{O}_{\mathrm{ss}} \rightarrow \mathrm{S}}}{\rightleftharpoons}}(\mathrm{S})
$$

Here, the various $k_{A \rightarrow B}$ are the rate constants of the different reactions. Although their accurate determination can be cumbersome, an estimate of the equilibrium constants can be obtained from the ratio of excitation/relaxation defined in eqn (5). Taking the energy quantum to the activation barrier from a given site (see Table 1) yields the associated classical, quasi-thermal activation probability, i.e.

$$
P_{A \rightarrow B}^{\ddagger}=\lim _{\hbar \omega_{i j} \rightarrow \Delta E_{A \rightarrow B}^{\ddagger}} R_{i j}
$$

The equilibrium constant is then estimated as the ratio of the forward to backward reaction, i.e.

$$
K_{A \rightarrow B}^{(\mathrm{eq})}=\frac{k_{A \rightarrow B}}{k_{B \rightarrow A}} \sim \frac{P_{A \rightarrow B}^{\ddagger}}{P_{B \rightarrow A}^{\ddagger}}
$$

The results are shown in Fig. 8. From the top two panels, it can be observed that the subsurface site exhibits a strong preference for resurfacing (dashed blue lines), for both the clean and $\Theta=3 / 4$ surfaces. This preference is more pronounced for the clean surface. Further, the bulk hydrogen tends also to migrate towards the second octahedral subsurface, albeit being somewhat less favorable than the subsequent diffusion to the subsurface from the second subsurface site. The attraction of the hydrogen towards the surface site is less important for the $\Theta=3 / 4$ surface, as indicated by the equilibrium constants slightly above one for the deeper octahedral sites. The situation for the fcc pre-covered surface is exactly the opposite, with equilibrium constants below one indicating a preference for diffusion towards the bulk. This is a clear indication that hydrogen atoms tend to repel each other once dissociated in the vicinity of the palladium surface. This same situation is also recognizable for the hcp pre-covered surface, whereas the hydrogen impurity seems to exhibit a slight preference for the second octahedral subsurface site. Interestingly, all equilibrium constants tend towards one at higher effective bias than the one shown in Fig. 8. Indeed, it is to be expected that, when the

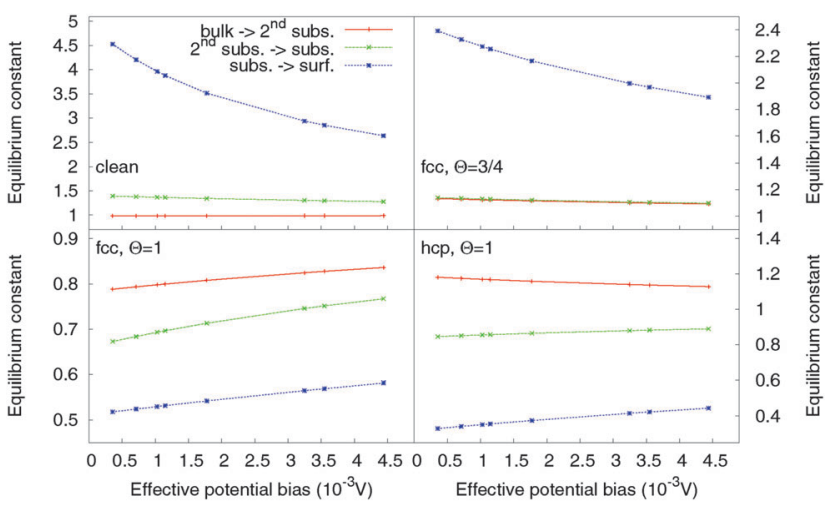

Fig. 8 Equilibrium constant, eqn (9), for the diffusion of an hydrogen impurity as a function of the effective potential bias for various surface coverages. The unsaturated surfaces (top panels) exhibit a strong preference for resurfacing. The fcc and hcp pre-covered surface have a preference for the diffusion to the bulk and the second surface octahedral sites, respectively. At large effective bias, all equilibrium constants tends asymptotically to one. 
potential term in eqn (5) dominates, the detailed balance will reach one and no net preference for the diffusion in either direction will be recognized. These observations point towards a strong tendency for the hydrogen impurity to resurface whenever possible before potentially occupying the region below the surface.

As discussed elsewhere, ${ }^{17}$ the population of the second octahedral subsurface site leads to an outward relaxation of the palladium surface and the hydrogen coating by about $0.1 \AA$. This is at the lower bound of the values consistent with experimental observations, 0.1-0.6 $\AA$, which would match better the palladium relaxation for the population of the subsurface site. In view of the current dynamical simulations, this hypothesis seems unviable since a bare surface will strongly attract any hydrogen stemming from the bulk. Simulations with hydrogens placed on the surface confirmed that no excitation of surface hydrogen directly to the subsurface is possible in the bias and current range studied here. This is in line with the equilibrium constant values shown in Fig. 8. It thus appears that the STMinduced protuberances observed experimentally are due to hydrogen clustering at and below the surface, after the surface has been saturated by neighboring hydrogen atoms.

\section{Conclusions}

In conclusion, we have presented dynamical simulations of the migration of a hydrogen impurity at a palladium interface upon excitation using scanning tunneling microscopy. The coupling of the impurity with the STM tip and with the electrons of the surrounding was treated using a recently introduced nonadiabatic coupling model. The model was extended to include the asymmetry of the projected density of states on the impurity around the Fermi energy. The effect of surface pre-coverage was investigated using newly available potential energy curves that include surface relaxation along a minimal energy reaction path spanning the surface to the third octahedral site below the surface.

It is found that, even at low potential bias or STM current, the hydrogen impurity invariably prefers resurfacing in all cases where the surface is not saturated. This is consistent with the experimental findings and the static DFT calculations at finite potential bias of Mitsui et al. ${ }^{16}$ Depending on the patterning of saturated surfaces, the hydrogen impurity migrates within a few tens of nanosecond to the most thermodynamically stable species, which is the second subsurface octahedral site in the hcp pre-covered case, for example. The transfer efficiency from the second surface octahedral site is found to be small in all simulations for saturated surfaces. This is in great contrast with simulations initiated in the subsurface site, which exhibit an almost quantitative population loss. For the unsaturated surface, the depopulation of the sites below the surface is also quantitative, as the hydrogen impurity simply migrates to the surface.

The subsurface remains in all cases practically unoccupied at longer times, which contradicts the hypothesis that the experimentally observed protrusions are due to migration of bulk hydrogens to the subsurface. ${ }^{14}$ The protuberances are most likely due to the clustering of hydrogens in the vicinity of the surface, with the topological feature mostly due to a hydrogen layer at the surface and to the partial population of the subsurface sites. This type of structures has also been inferred from experimental observations. ${ }^{16}$

The dynamical simulations confirm that the system behaves thermodynamically in the long time limit upon STM excitation. The transfer time extracted from the simulation exhibits an exponential dependence on an effective potential bias that includes the STM current, the actual STM bias, and the number of available states per palladium atoms. The transfer times extracted from the simulations show a similar asymmetry with respect to the potential bias sign as the non-adiabatic coupling rates, albeit about two orders of magnitude slower. The quasithermal nature of the excitation mechanism is highlighted by the evaluation of the equilibrium constants from the rates. As expected from the dynamical simulations, the equilibrium constants indicates a strong preference towards resurfacing whenever possible, while accumulation at the second subsurface octahedral or even deeper below is observed for the hcp and fcc pre-covered surfaces, respectively. This is, there is a repulsive interaction between surface hydrogen atoms and deeper hydrogen atoms. It must be noted that the small differences in the diffusion barriers below saturated surfaces are within the uncertainty of the calculated potential energy surfaces.

Future investigations will aim at understanding the role played by lateral diffusion on hydrogen clustering at the $\operatorname{Pd}(111)$ interface for various pre-coverages in order to explain the exact mechanism behind the experimental results. ${ }^{14,16}$

\section{Acknowledgements}

Financial support by the Gobierno Vasco-UPV/EHU project IT756-13, the Spanish Ministerio de Economía y Competitividad (Grant No. FIS2013-48286-C02-02-P), and the Deutsche Forschungsgemeinschaft through the Emmy-Noether program (project Tr1109/2-1) is acknowledged. M.B.-R. acknowledges the European Commission (Grant No. FP7-PEOPLE-2010-RG276921).

\section{References}

1 C. Chen, Introduction to Scanning Tunneling Microscopy, Oxford Univ. Press, New York, 1993.

2 Scanning Probe Microscopy and Spectroscopy: Theory, Techniques, and Applications, ed. D. Bonnel, Wiley, New York, 2001.

3 A. M. Moore and P. S. Weiss, Annu. Rev. Anal. Chem., 2008, 1, 857-882.

4 C. Stipe, M. A. Rezaei, W. Ho, S. Gao, M. Persson and B. I. Lundqvist, Phys. Rev. Lett., 1997, 78, 4410.

5 J. R. Hahn and W. Ho, Phys. Rev. Lett., 1997, 78, 4410.

6 J. Henzl, M. Mehlhorn, H. Gawronski, K.-H. Rieder and K. Morgenstern, Angew. Chem., Int. Ed., 2006, 45, 603.

7 M. Alemani, M. V. Peters, S. Hecht, K.-H. Rieder, F. Moresco and L. Grill, J. Am. Chem. Soc., 2006, 128, 14446-14447. 
8 K. Morgenstern, Acc. Chem. Res., 2009, 42, 213-223.

9 J. I. Pascual, N. Lorente, Z. Song, H. Conrad and H.-P. Rust, Nature, 2003, 423, 525-528.

10 T. Komeda, Prog. Surf. Sci., 2005, 78, 41.

11 M. Schmid, S. Crampin and P. Varga, J. Electron Spectrosc. Relat. Phenom., 2000, 109, 71-84.

12 M. Rose, A. Borg, T. Mitsui, D. Ogletree and M. Salmeron, J. Chem. Phys., 2001, 115, 10927.

13 G. H. Enevoldsen, H. P. Pinto, A. S. Foster, M. C. R. Jensen, W. A. Hofer, B. Hammer, J. V. Lauritsen and F. Besenbacher, Phys. Rev. Lett., 2009, 102, 136103.

14 E. C. H. Sykes, L. C. Fernández-Torres, S. U. Nanayakkara, B. A. Mantooth, R. M. Nevin and P. S. Weiss, PNAS, 2005, 102, 17907.

15 M. Blanco-Rey, M. Alducin, J. I. Juaristi and P. de Andres, Phys. Rev. Lett., 2012, 108, 115902.

16 T. Mitsui, E. Fomin, D. Ogletree, M. Salmeron, A. Nilekar and M. Mavrikakis, Angew. Chem., Int. Ed., 2007, 46, 5757.

17 M. Blanco-Rey and J. Tremblay, J. Chem. Phys., 2015, 142, 154704.

18 S. Gao, M. Persson and I. Lundqvist, Solid State Commun., 1992, 84, 271.

19 S. Gao, Phys. Rev. B: Condens. Matter Mater. Phys., 1997, 55, 1876.

20 S. Gao, M. Persson and I. Lundqvist, Phys. Rev. B, 1997, 55, 4825-4836.
21 J. C. Tremblay, J. Chem. Phys., 2013, 138, 244106.

22 J. P. Perdew, K. Burke and M. Ernzerhof, Phys. Rev. Lett., 1997, 78, 1396.

23 P. Giannozzi, S. Baroni, N. Bonini, M. Calandra, R. Car, C. Cavazzoni, D. Ceresoli, G. L. Chiarotti, M. Cococcioni, I. Dabo, A. D. Corso, S. de Gironcoli, S. Fabris, G. Fratesi, R. Gebauer, U. Gerstmann, C. Gougoussis, A. Kokalj, M. Lazzeri, L. Martin-Samos, N. Marzari, F. Mauri, R. Mazzarello, S. Paolini, A. Pasquarello, L. Paulatto, C. Sbraccia, S. Scandolo, G. Sclauzero, A. P. Seitsonen, A. Smogunov, P. Umari and R. M. Wentzcovitch, J. Phys.: Condens. Matter, 2009, 21, 395502.

24 H. J. Monkhorst and J. D. Pack, Phys. Rev. B: Solid State, 1976, 13, 5188.

25 K. Nobuhara, H. Nakanishi, H. Kasai and A. Okiji, J. Appl. Phys., 2000, 88, 6897.

26 K. Nobuhara, H. Nakanishi, H. Kasai and A. Okiji, J. Appl. Phys., 2002, 92, 5704.

27 N. Ozawa, T. A. Roman, H. Nakanishi, H. Kasai, N. B. Arboleda Jr. and W. A. Diño, J. Appl. Phys., 2007, 101, 123530.

28 J. C. Tremblay and T. Carrington Jr., J. Chem. Phys., 2006, 125, 094311.

29 J. C. Tremblay, S. Beyvers and P. Saalfrank, J. Chem. Phys., 2008, 128, 194709.

30 J. C. Tremblay, S. Monturet and P. Saalfrank, Phys. Rev. B, 2010, 81, 125408. 